\title{
Implications from the optical to UV flux ratio of Fe II emission in SDSS DR7 quasars
}

\author{
Hiroaki Sameshima* and Kimiaki Kawara \\ University of Tokyo (Japan) \\ E-mail: hsameshimadioa.s.u-tokyo.ac.jp, kkawaradioa.s.u-tokyo.ac.jp
}

We have investigated Fe II emission by analysing SDSS DR7 quasars. We have chosen those quasars which have both optical and UV Fe II emission simultaneously covered by the SDSS, resulting in 1,400 sources whose redshifts range from 0.73 to 0.80 and absolute i magnitudes from -24 to -26 . We found that narrow-line objects, such as Narrow Line Seyfert 1 galaxies (NLS1s), have large optical-to-UV Fe II flux ratios, indicating that they have large column-density Broad Line Region (BLR) clouds [ [ 9 ]. Combining this result with high Eddington ratios found in NLS1s, it can be naturally interpreted that the radiation pressure of photons emitted from the central source controls the state of BLR clouds. We also find that Fe II emission must emerge from the outermost region of BLR clouds in such a way that they are not radiatively blown away. Finally, we introduce our project to measure the iron abundance in BLR clouds by combining X-ray with optical observations.

Narrow-Line Seyfert 1 Galaxies and their place in the Universe April 4-6, 2011

Milano, Italy

\footnotetext{
* Speaker.
} 


\section{Introduction}

It is well known that the strength of the Fe II emission, which is one of the most intriguing characteristics of NLS1s, varies largely (especially in the optical) from object to object . Figure $\square$ shows the simplified Grotrian diagram of Fe II. The optical Fe II emission is emitted only by the 3-2 transition. However, since the branching ratio of 3-1 to 3-2 transition is about 1000:1, strong optical Fe II emission requires large scattering (more than 1,000 times) for the Fe II photon emitted by the 3-1 transition. Thus it is expected that the optical to UV flux ratio of Fe II emission can be used as the column density indicator. Figure $\square$ shows the results of numerical simulation of Fe II emission performed by [四]. In the following, we will estimate the column density of BLR clouds of SDSS DR7 quasars using Joly's relation.

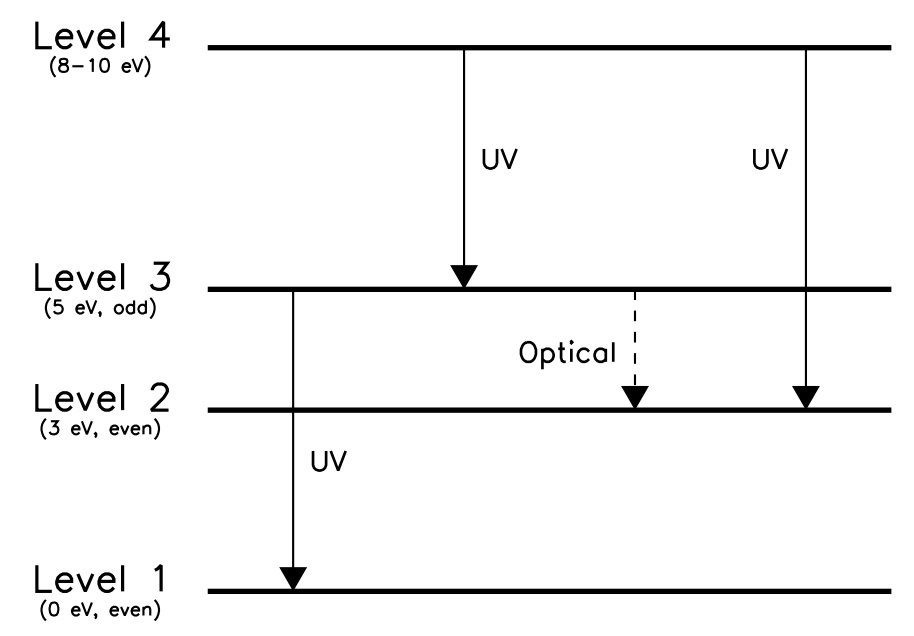

Figure 1: Simplified Fe II Grotrian Diagram

\section{Measurement}

To measure the UV and optical Fe II emission lines simultaneously, we have selected spectra which cover the wavelength range from 2200 to $5100 \AA$ in the rest frame, corresponding to the redshift range from 0.727 to 0.804 , from SDSS DR7 quasars. We then checked the signal-to-noise ratio $(\mathrm{S} / \mathrm{N})$ per pixel for each spectrum which fulfills median $\mathrm{S} / \mathrm{N}>10$ per pixel at their continuum levels for accurate flux measurements. Our final sample thus consists of 1,452 spectra.

Fe II emission lines are measured by template fitting. We adopt the Fe II template provided by [[]]. Figure [3] show an example of our fitting result. The black hole masses are estimated from the luminosity and the line width of $\mathrm{Mg}$ II.

\section{Results}

Figure $\mathrm{G}$ shows the relation between $\mathrm{Fe} \mathrm{II}(\mathrm{opt}) / \mathrm{Fe} \mathrm{II}(\mathrm{UV})$ and the Eddington ratio. A positive correlation is seen. Linear regression analysis, using an IDL procedure 'FITEXY.pro' (cf. 


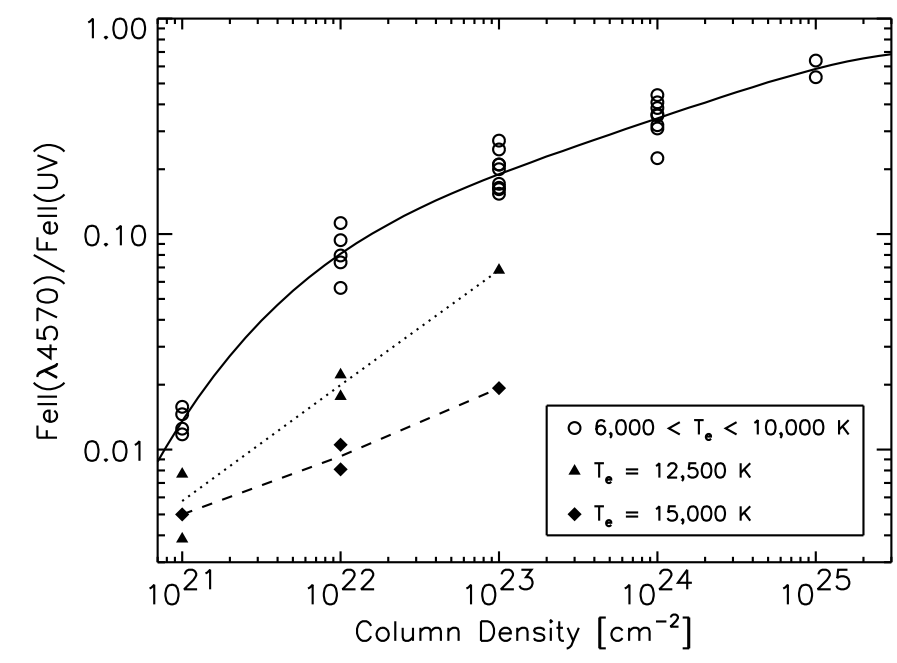

Figure 2: Fe II(opt)/Fe II(UV) vs. column densities from [U]

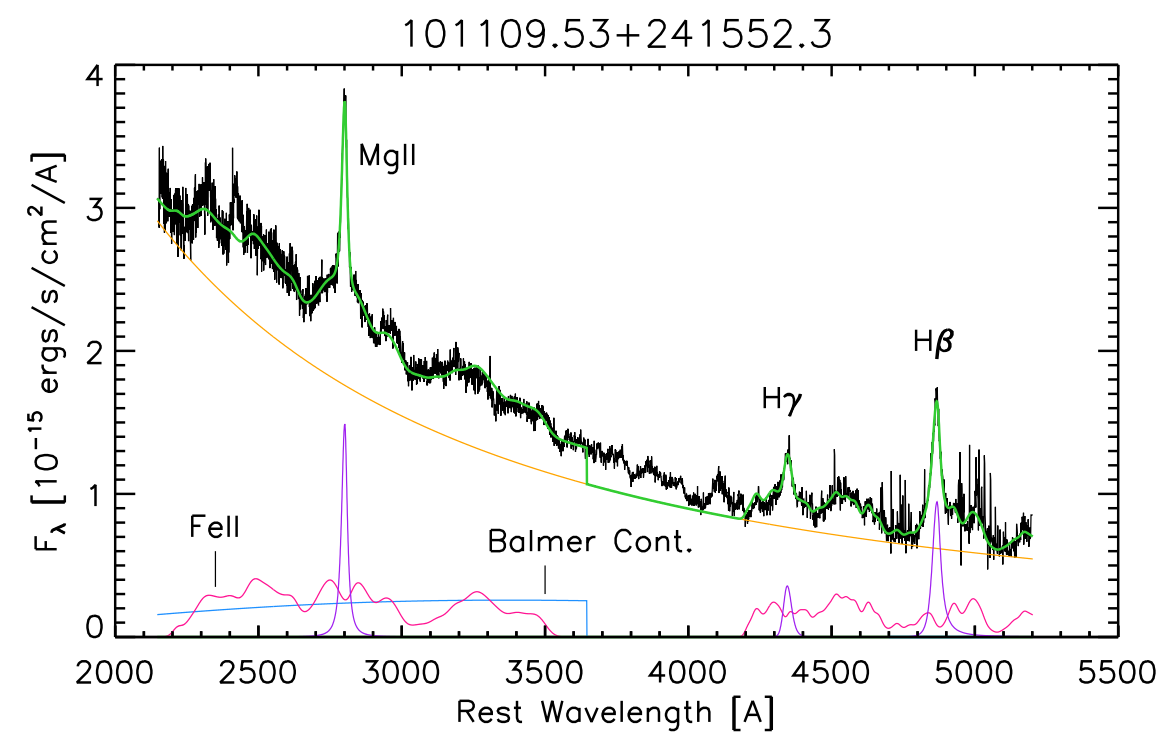

Figure 3: An example of Fe II fitting

[B] $)$, gives the relation as $\log F e I I(o p t) / F e I I(U V)=-0.55+0.39 \log L_{b o l} / L_{E d d}$. Spearman's rank correlation coefficient for assessing the non-linear correlation is $r_{S}=0.47$. This means that the probability of the null hypothesis that there is no correlation is less than $10^{-13}$. Thus, the correlation between $\mathrm{Fe}$ II(opt)/Fe II(UV) and the Eddington ratio is real. This implies that the column density increases with the Eddington ratio, because Fe II(opt)/Fe II(UV) increases with the column density.

Red star symbols indicate the sample included in the NLS1 catalog compiled by [四. As can be seen, NLS1s have large Eddington ratios and large optical to UV flux ratios of Fe II. 


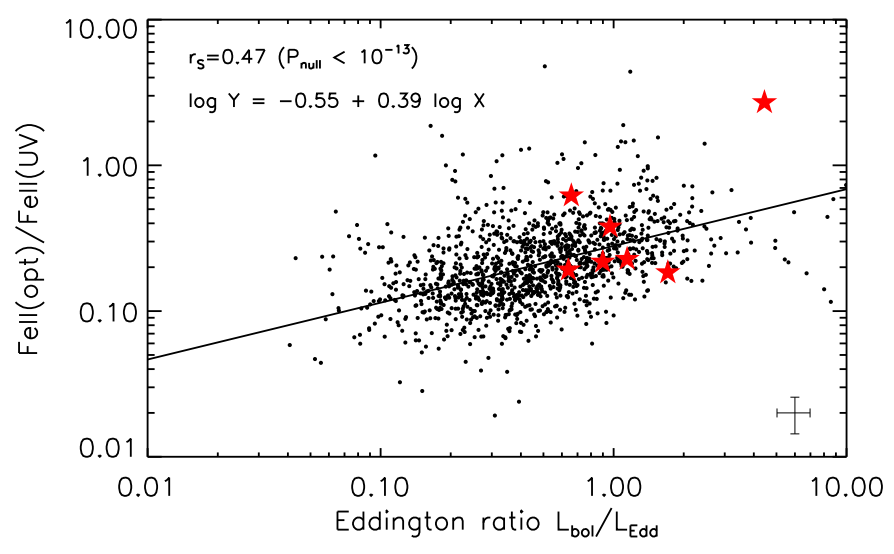

Figure 4: Fe II(opt)/Fe II(UV) vs. Eddington Ratio

\section{Discussion}

Combining the observed Fe II(opt)/Fe II(UV) and Joly's result shown in Fig. 凹, we estimated column densities. The obtained column density distribution is shown in Figure [5]. [D] claimed that radiation pressure does play an important role in BLR gas dynamics if the column density distribution of AGNs have intrinsic dispersion. Fig. [ supports [వ], that is, radiation pressure does play an important role in BLR gas.

In the situation that radiation pressure does play an important role in BLR gas, the traditional Eddington ratio must be corrected. Figure $\mathbf{B}$ shows the relation between the column density (estimated from Fe II emission lines) and the Eddington ratio. Each line represents where the corrected Eddington ratio become unity, so that the lower region of the lines corresponds to a super-Eddington area. The coefficient a represents the ionizing photon (i.e. $h v>13.6 \mathrm{eV}$ ) to the incident photon ratio. If we adopt $a=0.6$, which is an average value for an AGN BLR cloud (cf. []]), almost all of our samples become super-Eddington, resulting in significant radiation-pressure-driven winds. To overcome this super-Eddington problem, Fe II emission lines must emerge from the outermost region of BLR clouds where ionizing photons are diluted enough that $a<0.01$.

\section{Future Work}

In the above discussion, one important assumption is made, that the abundance of the BLR cloud is fixed to be solar. If the iron abundance of a BLR cloud is different than solar, the lower limit of the column density of the BLR cloud needed to not be blown away by radiation pressure also becomes different. In other words, if we are able to measure independently the (Hydrogen) column density of the BLR clouds, we can estimate the iron abundance from the optical to UV flux ratio of Fe II emission lines.

To measure the Hydrogen column density, an X-ray observation is the most suitable one. In the X-ray region, incident photons on the BLR clouds are eaten by photoelectric absorption. Figure $\square$ simulates how an incident SED is changed by photoelectric absorption of BLR clouds with 


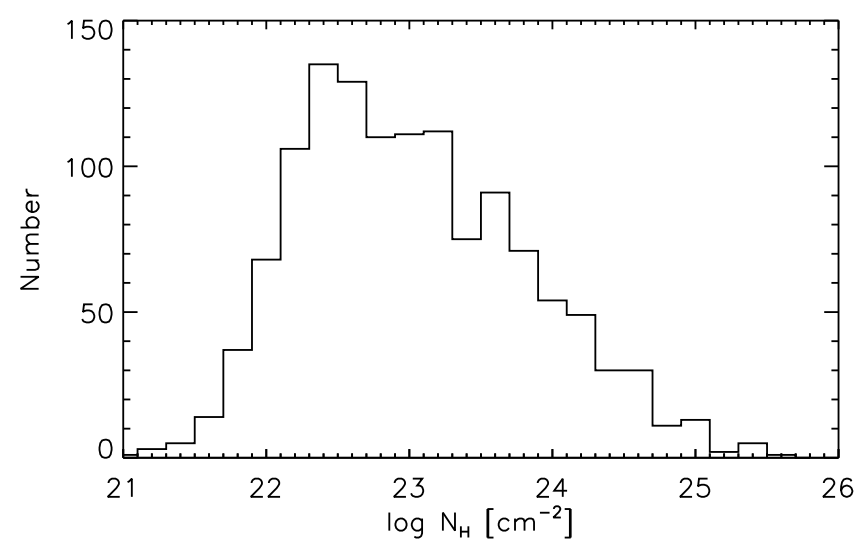

Figure 5: Column density distribution

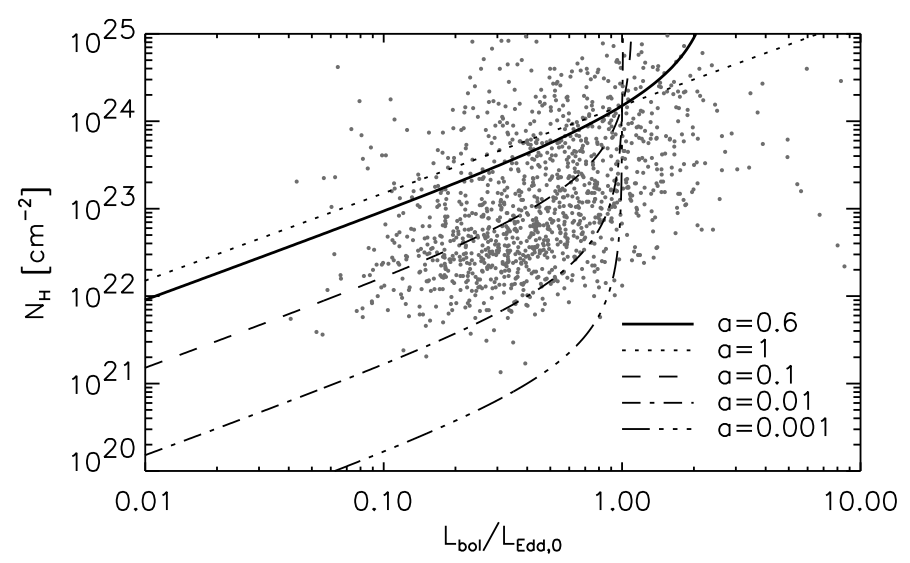

Figure 6: Estimated column density vs. Eddinton ratio

$N_{H}=10^{23}, 10^{24} \mathrm{~cm}^{-2}$. We submitted a proposal to X-ray satellite "SUZAKU", which is recently accepted. We now planning to estimate iron abundances of I Zw1, PG 1322+659 and PG 1626+554.

\section{References}

[1]M. Joly, Formation of low ionization lines in active galactic nuclei, 1987, A\&A, 184, 33

[2]A. Marconi, D.J. Axon, R. Maiolino, T. Nagao, P. Pietrini, G. Risaliti, A. Robinson and G. Torricelli, On the Observed Distributions of Black Hole Masses and Eddington Ratios from Radiation Pressure Corrected Virial Indicators, 2009, ApJ, 698, L103

[3]W.H. Press, S.A. Teukolsky, W.T. Vetterling and B.P. Flannery, Numerical Recipes, Cambridge Univ. Press, 1992

[4]H. Sameshima, K. Kawara, Y. Matsuoka, S. Oyabu, N. Asami and N. Ienaka, Implications from the optical to ultraviolet flux ratio of Fe II emission in quasars, 2010, MNRAS, 410, 1018

[5]T. Tsuzuki, K. Kawara, Y. Yoshii, S. Oyabu, T. Tanabe and Y. Matsuoka, Fe II Emission in 14 Low-Redshift Quasars. I. Observations, 2006, ApJ, 650, 57 


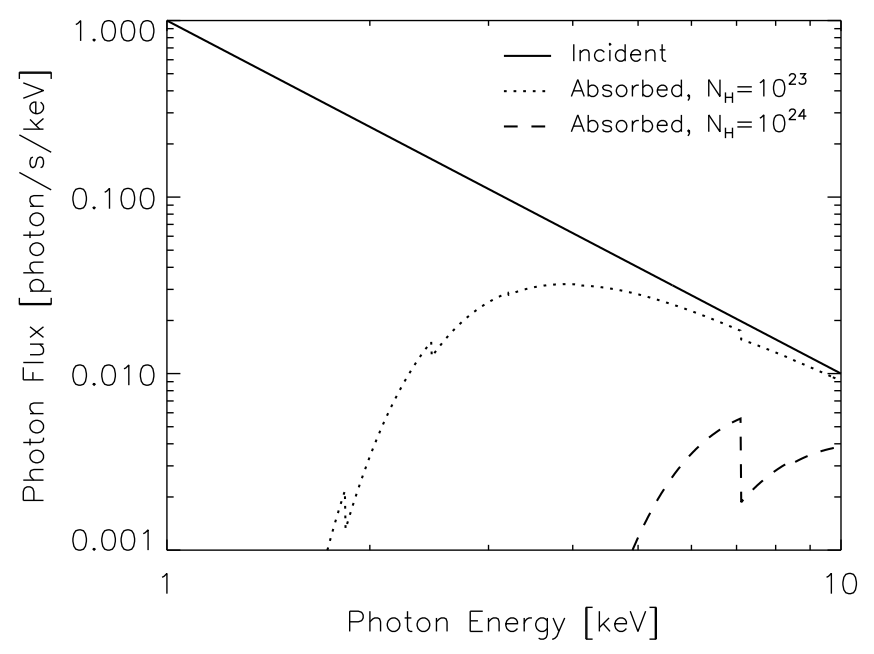

Figure 7: Photoelectric absorption of BLR gas

[6]H. Zhou, T. Wang, W. Yuan, H. Lu, X. Dong, J. Wang and Y. Lu, A Comprehensive Study of 2000 Narrow Line Seyfert 1 Galaxies from the Sloan Digital Sky Survey. I. The Sample, 2006, ApJS, 166, 128 